\title{
Application Analysis of Electrical Automation in Electrical Engineering
}

\section{Xianglong Liu Shengke Hu}

Hubei Power Transmission and Transformation Engineering Co., Ltd., Wuhan, Hubei, 430000, China

\begin{abstract}
Electrical automation technology is widely used in today's electrical engineering, but with the improvement of electrical engineering construction requirements, the application of electrical automation technology also needs to be promoted and reformed. Electrical automation technology can not only enhance the safety and stability of electrical engineering operations, but also improve the overall working efficiency of engineering projects, so it has been recognized and widely used in more and more electrical engineering fields. Based on this, the paper discusses the practical application of electrical automation technology in electrical engineering, and discusses the shortcomings.
\end{abstract}

\section{Keywords}

electrical automation; electrical engineering; application

\section{电气自动化在电气工程中的应用分析}

\section{刘湘龙 胡生科}

湖北送变电工程有限公司，中国・湖北 武汉 430000

\section{摘 要}

电气自动化技术在现如今的电气工程当中被广泛应用, 但随着电气工程施工要求的提升, 电气自动化技术的应用也需要进行 全方位的提升和改革。电气自动化技术不仅可以使电气工程作业安全性和稳定性得到增强, 还能够使工程项目的整体工作效 率得到提高, 所以已经被越来越多的电气工程领域认可并进行广泛应用。基于此, 论文针对电气自动化技术在电气工程当中 的实际应用进行探讨，并对所存在的不足之处进行论述。

\section{关键词}

电气自动化; 电气工程; 应用

\section{1 引言}

信息自动化技术在电气工程领域当中的应用是不可避免 的, 对于中国的电气企业来讲, 将电气自动化技术在中国电 气工程施工当中进行广泛应用, 在发展过程中也顺应了整个 世界的发展潮流。但在实际应用过程中仍然存在着一些问题 与不足需要进行解决, 从而推动中国电气工程领域的全面进 步。论文对项目当中的不足之处进行分析, 然后提出有效建 议与对策, 使电气自动化技术在中国电气工程领域当中的应 用效果得到提高 ${ }^{[1]}$ 。

【作者简介】刘湘龙 (1990-), 男, 中国湖南长沙人, 本科学历, 电气工程师, 从事电气工程及其自动化研究。

\section{2 电气自动化技术的必要性}

2.1 电气自动化技术的应用将使中国电气工程智能化 建设水平得到不断提升

在中国综合国力以及经济水平不断提升的过程中, 科学 技术的发展与进步也非常显著, 正在不断朝向现代化和智能 化的方向进行发展。智能化的发展方向是指在未来机械设备 可以依照人本身的意愿从事自动化的活动, 大大解放施工作 业时人力的投入。因此，在电气工程当中，智能化技术和设 备的广泛应用是未来的必然趋势。

\section{2 能够对电力系统开展全方位的有效监控}

电气工程是人们日常生活与生产过程中重要的组成部分 之一, 如果电气工程的系统运行受到了影响或者是出现了故 
障问题, 那么人们的生活以及工作都会受到严重威胁。因此, 需要通过电气自动化技术及相关设备开展全方位的实时监控, 针对电力系统的运行安全性以及稳定性进行保障, 及时发现 存在的故障隐患并及时处理 ${ }^{[2]}$ 。

\section{3 电气自动化技术应用存在的问题}

\section{1 节能效果并不好}

对于电气自动化生产技术来讲, 在实际应用过程中主要 是对电气工程进行控制, 但在控制过程中所产生的节能效果 并不是非常理想, 这也是当前电气自动化技术应用存在的主 要问题之一。

首先, 在电力系统当中, 输电线路本身电阻是不能够完 全消除的，所以会导致节能受到一定程度的影响。

其次, 电流在传输的过程中会导致输电线路发热而产生 不必要的能耗, 尤其是对于建筑物内的电气工程来讲, 这一 问题是最为显著的。

最后, 中国许多建筑物内部的居住用户会使用多种用电 设备, 并且是在同一时间, 所以建筑物内的售电线路布局非 常的复杂, 因此所产生的能耗问题也变得更加严重 ${ }^{[3]}$ 。

\section{2 易受影响}

对于电气自动化设备来讲, 在实际应用的过程中会受到 外界环境温度以及湿度等其他影响因素的干扰, 这也是电气 自动化设备无法避免的一项问题。因为对于大多数的电气自 动化设备来讲, 需要在特定的环境条件下进行应用, 如果环 境条件被破坏, 那么电气自动化设备的应用情况以及效果都 会受到影响。例如, 对于一些特定的电气自动化设备来讲, 如果运行环境的温度过高, 那么可能会导致设备直接失效或 者是出现㿈疾现象, 因此在实际应用的过程中, 需要对设备 使用环境进行全方位的监控。但是, 这项工作却导致电气自 动化设备的使用难度大大增加, 还增加了一些不必要的人力 资源支出和经济支出 ${ }^{[4]}$ 。

\section{4 电气工程中电气自动化的应用}

\section{1 电网调度中电器自动化技术的应用}

对于电力系统来讲, 电网调度最主要的目的是使整个系 统实现安全、稳定的运行, 并面向社会用户提供更加稳定的 能源供应。在此过程中, 电器自动化技术的应用主要是对整 个系统运行过程中设备的数据进行采集, 然后再根据电网的
实际运行参数和电网控制系统的要求, 对控制指令进行不断 调整, 从而使供电的可靠性得到增强。在整个电网调度工作 当中, 电气自动化技术所发挥的作用是不可替代的, 因为随 着当前社会的进步与发展, 用电量的需求也在不断增加, 所 以中国建设电网的覆盖面积也在不断增加, 电网调度工作的 难度随之提高。

在应用电气自动化技术的过程中, 可以使整个电力系统 的信息数据采集工作能力得到提升, 还可以开展实时的监控 与评估, 使电力系统的运行状态能够始终处于掌控当中。相 关调度工作部门可以根据所反馈的监控以及评估结果, 让调 度中心下达相应的指令, 使各项指令的下达都拥有准确的判 断依据 ${ }^{[5-6]}$ 。

\section{2 变电站工作中电气自动化技术的应用}

在电力系统当中, 变电站的主要作用是对电压进行转换 以及接受和分配电能, 同时还能够对电力流向以及电压等进 行调整和控制, 属于整个电力系统当中最重要的设施之一。 对于电网建设来讲, 变电站是不可缺少的一部分, 对于整个 电网的安全稳定运行具有非常重要的影响, 若是在实际运行 过程中变电站出现了相应的故障问题, 轻则导致供电稳定性 受到影响, 重者会导致大面积停电, 对社会的生产以及生活 都造成非常严重的影响。因此, 在电力系统运行过程中, 为 了使变电站的运行稳定性得到保障, 需要对变电站的运行状 态开展实时有效监控。在传统的监控工作中, 主要是以人力 为监控工作主体, 所取得的实际工作效率比较低。在电气自 动化技术应用的过程中, 将变电站的监控工作变成了远程监 控, 所以监控的时效性得到全面的增强, 并且在实际监控工 作当中, 人力成本的支出大大降低, 能够缓解人工作业压力 不断增加的问题。

采用电气自动化技术, 可以利用当前电力系统中的各变 电站设备开展远程监控, 对设备的运行状态作出全方位的评 估。当设备出现相应故障问题时, 可以及时进行报警, 使变 电站设备的维修工作具有稳定基础。因此, 对于电力系统的 变电站工作来讲, 应用电气自动化技术实现了变电站的多层 次以及全方位监控, 而且通过网络化以及智能化的管理手段, 使变电站的整体管理工作效率以及工作水平都得到了提高, 大大延长了变电站设备的使用寿命, 同时降低了变电站设备 维护与保养工作中所投入的成本, 对于变电站的自动化建设 
工作来讲是具有重要意义的。

\section{3 继电保护器中的电气自动化技术应用}

继电保护器是当前电气工程中最重要的组成部分之一, 能够对电气设备的运行状态开展实时监测, 也是在出现故障 问题时进行及时报警的主要部件, 所以在电气工程当中, 继 电保护器的作用是不可取代的。此外, 还能在实际应用过程 中为电气维修工作提供更多更准确的信息, 增加维修工作效 率, 并降低设备出现故障的几率。对于继电保护器来讲, 在 使用过程中会出现两种现象, 分别是误动和拒动, 工作人员 在监测以及运行调度过程中, 会被继电保护器的这两种错误 动作所引导, 不能及时针对电气设备进行保护处理。但在应 用电气自动化技术之后, 继电保护器的自动化处理工作水平 会得到极大程度提升, 如果电气设备本身出现了超负荷运行 的情况, 那么继电保护器不仅仅会发出预警通知, 而且还会 及时切断整个电气设备运行电路, 使其他电气设备的安全得 到保障。所以, 继电保护器当中应用电气自动化技术是非常 必要的, 它能够使整个电力系统的电气设备运行安全性得到 保障, 在出现故障问题时及时切断系统电源, 保障电力系统 以及电路运行安全。

\section{5 结语}

综上所述, 对于电气工程项目建设来讲, 随着经济的快 速进步与发展, 工程项目建设的要求也在随之提升。为了能
够使电气工程电力系统的供电稳定性和可靠性得到提高, 需 要在时代发展所提出的更高要求下, 对电气工程开展全方位 的现代化以及信息化建设工作。论文所论述的电气自动化技 术在电气工程中的应用, 就是为了对电气自动化技术在当前 电力系统电气工程中的应用进行探讨, 并对所存在问题进行 研究, 促进中国电气工程的进一步发展与建设水平提升, 使 中国经济的发展拥有坚实稳定的基础。因此，在今后的电气 工程项目建设过程中, 需要根据电气自动化技术的应用进行 全方位的探讨与研究, 使电气工程的自动化水平与智能化水 平得到全方位提高。

\section{参考文献}

[1] 刘大朋. 电气自动化技术在电气工程中的应用分析 [J]. 佳木斯教 育学院学报,2013(12):478.

[2] 张安国. 电气自动化技术在电气工程中的应用分析 [J]. 中国高新 技术企业 ,2016(13):52-53.

[3] 李猜. 关于电气自动化在电气工程中的应用分析 [J]. 河南科 技, 2014(04):122.

[4] 孙慧莹, 王恩双. 关于电气自动化在电气工程中的应用分析 [J]. 商 品与质量 $, 2015(18): 185$

[5] 韩金亭. 关于电气自动化在电气工程中的应用分析 [J]. 工程技术: 全文版,2016(09):208.

[6] 沈银环. 关于电气自动化在电气工程中的应用分析 [J]. 工程技术 : 文摘版 ,2016(12):55. 